\title{
Extrahepatic bile duct carcinoma with extensive intraepithelial spread: a clinicopathological study of 21 cases
}

\author{
Yoshitsugu Nakanishi ${ }^{1,2,3}$, Yoh Zen ${ }^{2}$, Hiroshi Kawakami ${ }^{4}$, Kanako Kubota ${ }^{3}$, Tomoo Itoh $^{3}$, \\ Satoshi Hirano ${ }^{1}$, Eiichi Tanaka ${ }^{1}$, Yasuni Nakanuma ${ }^{2}$ and Satoshi Kondo ${ }^{1}$ \\ ${ }^{1}$ Division of Cancer Medicine, Department of Surgical Oncology, Hokkaido University Graduate School of \\ Medicine, Sapporo, Japan; ${ }^{2}$ Department of Human Pathology, Kanazawa University Graduate School of \\ Medicine, Kanazawa, Japan; ${ }^{3}$ Department of Surgical Pathology, Hokkaido University Hospital, Sapporo, \\ Japan and ${ }^{4}$ Department of Gastroenterology, Hokkaido University Graduate School of Medicine, Sapporo, \\ Japan
}

\begin{abstract}
Extrahepatic bile duct carcinoma occasionally presents with intraepithelial spread for a considerable area around the main tumor. In this study, we compared clinicopathological features of extrahepatic bile duct carcinoma with and without extensive intraepithelial spread ( $\geq 20 \mathrm{~mm}$ from the main tumor). Out of 117 cases of extrahepatic bile duct carcinoma, 21 (18\%) were found to have extensive intraepithelial spread. Those cases were pathologically characterized by a papillary or nodular main tumor, a more differentiated histological grade, less deep invasion, and infrequent portal vein or hepatic invasion in comparison with cases without intraepithelial spread. Areas of intraepithelial spread histologically consisted of low-papillary growth (17 cases, $81 \%$ ) and completely flat growth (4 cases, $19 \%$ ) of carcinoma cells. The former histology corresponded to a macroscopic granular mucosa, whereas the latter growth was hardly detected by gross examination. Immunohistochemically, in 16 of 21 cases (76\%), at least one of p53, CEA, and MUC1 was expressed in both the main tumor and the spreading area. Interestingly, patients with intraepithelial spread had a better postoperative prognosis than those without intraepithelial spread $(P=0.009)$. However, three patients had anastomotic recurrence 54-130 months after surgery. In conclusion, intraepithelial-spreading bile duct carcinoma is characterized by papillary or nodular main lesions, a more differentiated histological grade, and less invasiveness. The presence of intraepithelial spread was not an indicator of a poor prognosis, but carcinoma in situ at the bile duct stump could cause late anastomotic recurrence after surgery. Modern Pathology (2008) 21, 807-816; doi:10.1038/modpathol.2008.65; published online 18 April 2008
\end{abstract}

Keywords: extrahepatic bile duct carcinoma; cholangiocarcinoma; superficial spread; carcinoma in situ

Macroscopic growth patterns of extrahepatic bile duct carcinoma can largely be classified into three types. The first is an infiltrating type showing diffuse sclerosis and thickening of the bile duct wall. The second is a nodular type showing a distinct nodular tumor. The last is intraluminal papillary growth. ${ }^{1,2}$ In addition, there is another growth pattern characterized by the intraepithelial spread of carcinoma cells. Intraepithelial spread influences the extent of, and sometimes results in incomplete, surgical resection. In 2004, Wakai et $a l^{3}$

Correspondence: Dr Y Nakanuma, MD, Department of Human Pathology, Kanazawa University Graduate School of Medicine, 13-1 Takaramachi, Kanazawa 920-8640, Japan.

E-mail: pbcpsc@kenroku.kanazawa-u.ac.jp

Received 20 January 2008; revised 02 March 2008; accepted 10 March 2008; published online 18 April 2008 reported that carcinoma in situ at the stumps of bile ducts might be responsible for late recurrence after surgery. In addition, we also reported two cases of late recurrence of extrahepatic bile duct carcinoma with intraepithelial spread after surgery. $^{4,5}$ However, the clinicopathological characteristics of extrahepatic bile duct carcinoma with intraepithelial spread have been barely reported. It has not been revealed whether or not intraepithelial-spreading bile duct carcinoma has unique pathological characteristics or intraepithelial spread impacts postoperative survival.

In this study, we examined clinicopathological differences between extrahepatic bile duct carcinoma with and without intraepithelial spread to elucidate clinicopathological characteristics of intraepithelial-spreading bile duct carcinoma. 


\section{Materials and methods}

\section{Patient Selection and Tissue Preparation}

We examined a total of 119 cases of surgically resected extrahepatic bile duct carcinoma in the Second Department of Surgery of Hokkaido University Hospital from December 1989 to February 2006. Two cases of flat carcinoma in situ without invasive lesions were excluded. Consequently, 117 cases of extrahepatic bile duct carcinoma were examined in the present study. The patients comprised 94 men and 23 women, and ranged in age from 34 to 84 years (mean, 66 years). The main tumor of these cases was located from the left or right hepatic duct to lower (intrapancreatic) extrahepatic bile duct. Tumors arising from the ampulla of Vater, cystic duct, and gallbladder were not examined in this study.

All of the surgically resected specimens were fixed in $10 \%$ buffered formalin. Serial sections at 3-6 mm intervals were prepared from the entire area of the extrahepatic bile duct. Histological specimens were similarly made from entire areas of hilar and intrahepatic large bile ducts in the patients who underwent concurrent hepatectomy. These sections were embedded in paraffin, and more than 20 sections were cut from each block. Several of them were processed routinely for histological study. The remaining sections were used for immunohistochemistry.

\section{Definition of Intraepithelial Spread of Carcinoma Cells}

We defined the intraepithelial spread of carcinoma as intraepithelial atypical cells similar to the cells composing the surface of the main tumor. Intraepithelial carcinoma cells had cellular atypia corresponding to biliary intraepithelial neoplasia-3 (BilIN-3, carcinoma in situ) ${ }^{6}$ Premalignant lesions corresponding to BilIN-1 or BilIN-2 were not considered intraepithelial carcinomas. ${ }^{6}$ The length of the intraepithelial spread was measured from the edge of the main tumor (Figure 1). In cases associated with several invasive lesions, the largest one was regarded as the main tumor. In this study, extensive intraepithelial spread was defined as being $\geq 20 \mathrm{~mm}$ in length from the margin of the main lesion to one side (proximal or distal).

\section{Clinicopathological Examination of the Main Tumor}

The main tumor of extrahepatic bile duct carcinoma was grossly classified into three types: papillary, nodular, and infiltrating. The papillary type had an evident papillary tumor showing a cauliflower-like appearance. The nodular type showed a discrete tumor abruptly elevated from the surrounding mucosa. Papillary and nodular types had evident tumorous lesions, whereas the infiltrating type showed a gradually and slightly elevated lesion. Thirteen clinicopathological features (gender, age, location of the main tumor, depth of invasion of main tumor (pT), lymph node metastasis $(\mathrm{pN})$, distant metastasis (pM), histological type, portal vein invasion, hepatic invasion, pancreatic invasion, venous invasion, lymphatic vessel invasion, and perineural invasion) were compared between extrahepatic bile duct carcinoma with and without extensive intraepithelial spread. Scoring of pT, pN, and pM was made according to the UICC system. ${ }^{7}$ Locations of main tumors were classified as either the distal (intrapancreatic) or proximal (extrapancreatic) bile duct. Histological grades were classified into well, moderately, and poorly differentiated adenocarcinoma, and other special types. Papillary adenocarcinoma was classified as well differentiated.

\section{Immunohistochemistry}

Immunostainings of p53, carcinoembryonic antigen (CEA), mucin core protein 1 (MUC1), and mesothelin were performed using the EnVision + system (Dako Cytomation, Glostrup, Denmark) in cases with extensive intraepithelial spread. After microwave treatment in $10 \mathrm{mM}$ citrate buffer ( $\mathrm{pH}$ 6.0) for $20 \mathrm{~min}$ (sections for p53, CEA, and mesothelin) and blocking of endogenous peroxidase and incubation in serum-free Protein Block Solution (Dako Cytomation), the deparaffinized and rehydrated sections were incubated overnight at $4^{\circ} \mathrm{C}$ with primary monoclonal antibodies: anti-p53 monoclonal antibody (clone DO-7; 1:100; Dako Cytomation), antiCEA monoclonal antibody (clone $\alpha-7$; $1: 100$; Dako Cytomation), anti-MUC1 monoclonal antibody (antiCA15-3; clone DF3; 1:50; Toray Fuji Bionics, Tokyo, Japan), and anti-mesothelin monoclonal antibody (clone 5B2; 1:20; Novocastra Laboratories, Newcastle, UK). The sections were then incubated at room temperature for $1 \mathrm{~h}$ with anti-mouse immunoglobulins conjugated to a peroxidase-labeled dextran polymer (EnVision + ; Dako Cytomation). The resection products were developed by immersing the section in a 3,3'-diaminobenzidine tetrahydrochloride (DAB) solution containing $0.03 \%$ hydrogen peroxide. Nuclei were lightly counterstained with hematoxylin.

The expression of p53, CEA, MUC1, and mesothelin was evaluated as positive or negative, according to the percentage of positive cells in the main tumor or extensive intraepithelial spread area: positive, more than $10 \%$, negative, less than $10 \%$.

\section{Postoperative Survival}

Follow-up information after surgical resection was available for all 117 patients. The mean follow-up period was 28 months (range, 1-170 months). We 


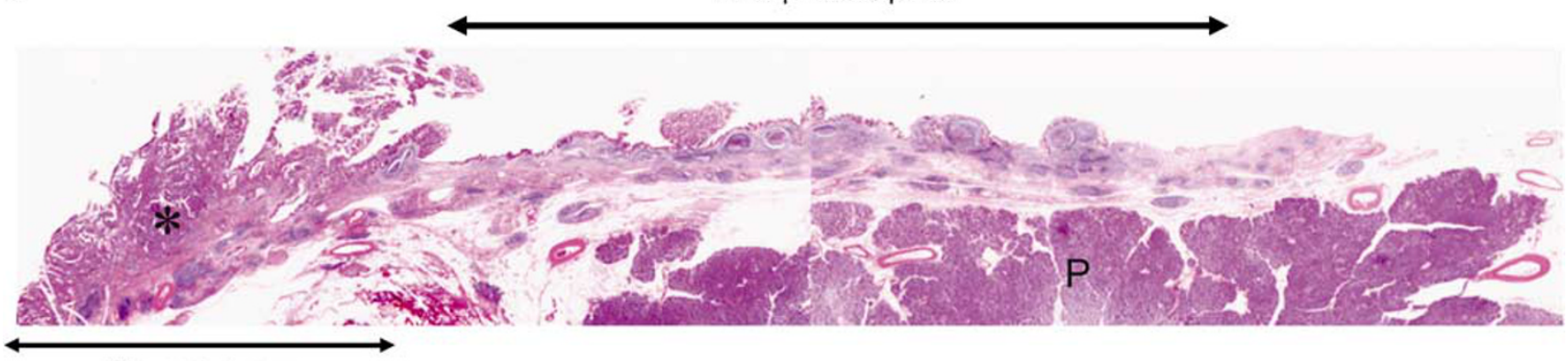

The main tumor

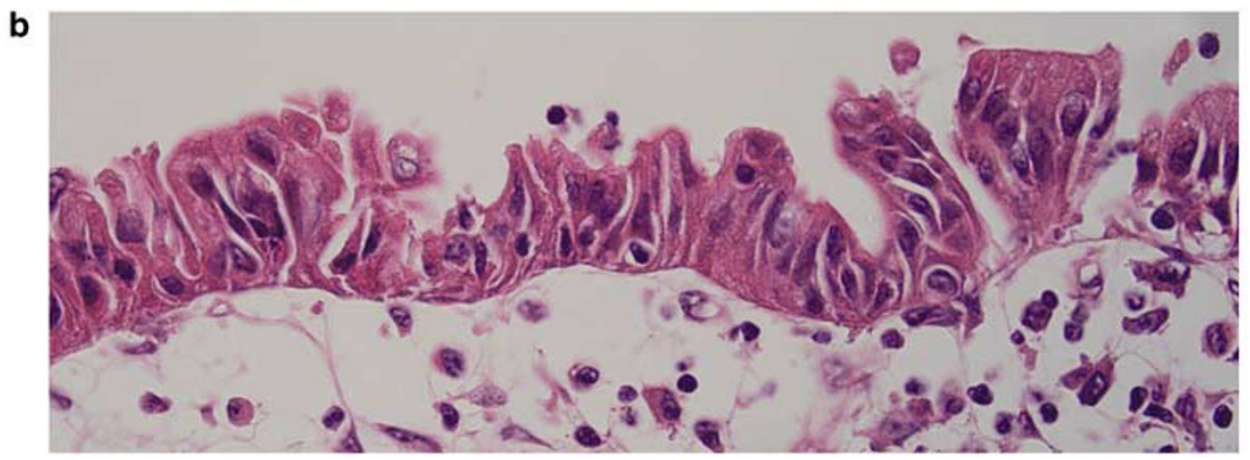

Figure 1 The representative appearance of extensive intraepithelial spread of extrahepatic bile duct carcinoma. (a) Loupe view of intraepithelial spreading of carcinoma cells in a case of extrahepatic bile duct carcinoma. The main tumor (*) is a papillary adenocarcinoma. P, pancreas. (H\&E). (b) Histological image of intraepithelial spreading of carcinoma. Intraepithelial carcinoma cells show enlarged nuclei, an irregular nuclear membrane, and a loss of cellular polarity. (H\&E, original magnification $\times 400$ ).

compared the postoperative survival of patients of extrahepatic bile duct carcinoma with and without extensive intraepithelial spread.

\section{Statistics}

A statistical analysis was performed using the $\chi^{2}$-test for independent variables or Fisher's exact test to examine differences of clinicopathological features between carcinoma with and without extensive intraepithelial spread. Survival curves were calculated by the Kaplan-Meier method, and differences in survival were evaluated using the log-rank test. A probability of $P<0.05$ was considered to be statistically significant.

\section{Results}

\section{Comparison of Clinicopathological Features Between Extrahepatic Bile Duct Carcinoma with and without Extensive Intraepithelial Spread}

The length of the intraepithelial spread of carcinoma cells from the edge of the main tumor is shown in Figure 2. Forty-six of one hundred and seventeen cases $(39 \%)$ were found to have intraepithelial spread. In 21 cases (18\% of all cases), the spread was $\geq 20 \mathrm{~mm}$ long.

Twenty-one patients with extensive intraepithelial spread consisted of 19 males and 2 females, and their ages ranged from 34 to 84 (mean, 65) years. There was no significant difference in age and gender among cases with and without extensive intraepithelial spread (Table 1). In extrahepatic bile duct carcinoma with extensive intraepithelial spread, main tumors were located in the distal (intrapancreatic) bile duct in 8 cases $(38 \%)$ and the proximal extrahepatic bile duct in 13 cases (62\%). Proximal cases included carcinoma in the right hepatic duct (two cases), left hepatic duct (two cases), and bifurcation of hepatic ducts (one case). The main tumors of intraepithelial-spreading bile duct carcinoma were more frequently located in the distal portion in comparison with carcinoma without extensive intraepithelial spread $(P=0.008)$. Main tumors of intraepithelial-spreading bile duct carcinoma macroscopically appeared to be the papillary type in 11 cases (52\%), nodular type in 8 cases $(38 \%)$, and infiltrating type in 2 cases $(10 \%)$. Intraepithelial-spreading bile duct carcinoma more frequently had papillary and nodular main tumors than carcinoma without extensive intraepithelial spread $(P<0.001)$. Histological types of main tumors were well-differentiated ( 12 cases, $57 \%$ ), moderately differentiated (7 cases, 33\%) and poorly differentiated adenocarcinoma (one case, $5 \%$ ). The remaining case $(5 \%)$ was adenosquamous carcinoma. Welldifferentiated adenocarcinoma was more common in cases with extensive intraepithelial spread than without extensive intraepithelial spread $(P=0.005)$. The depths of invasion of main tumors corre- 

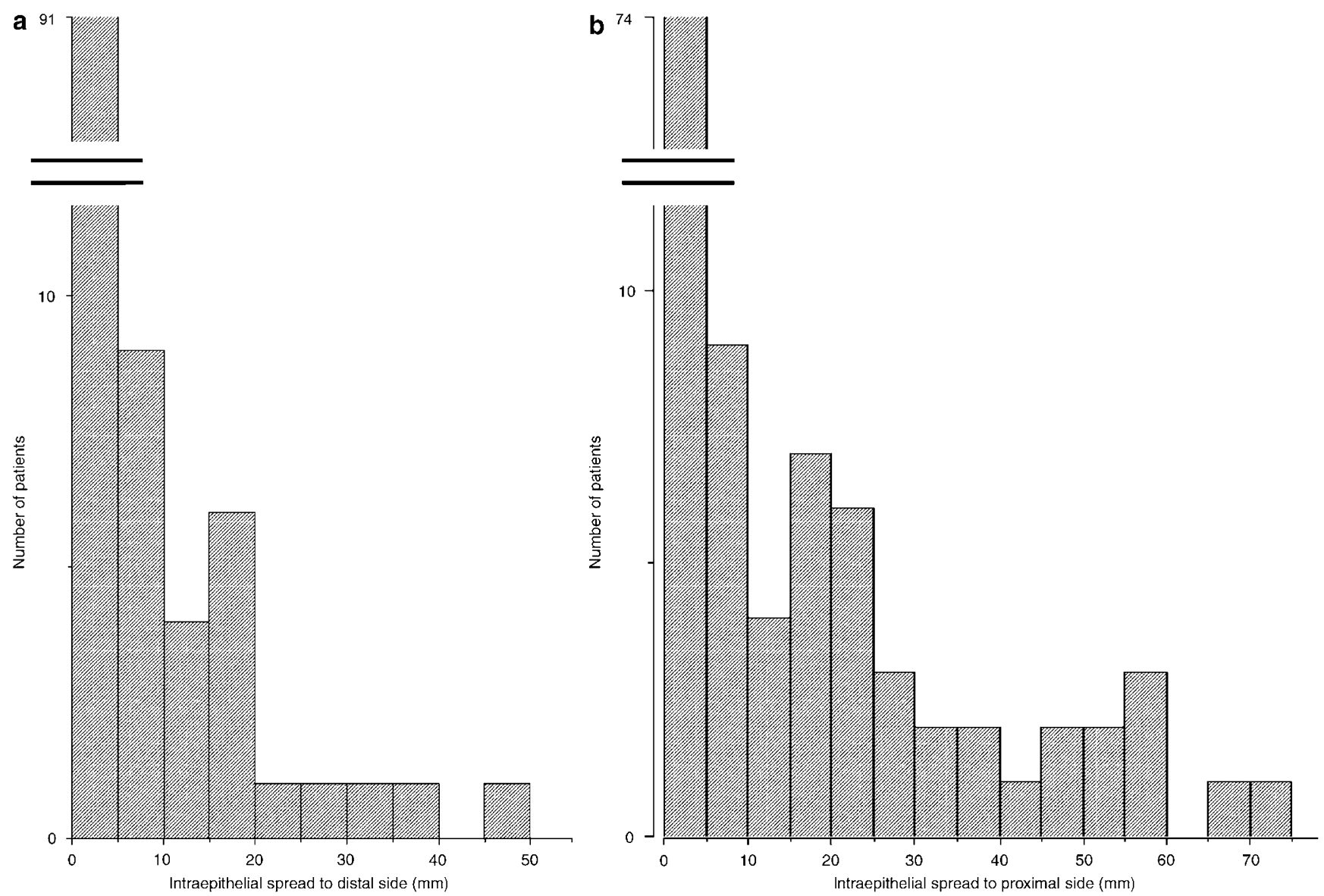

Figure 2 Length of intraepithelial spread from main tumors. (a) Distal side from main tumors. (b) Proximal side from main tumors.

sponded to pT1 in 3 cases (14\%), pT2 in 10 cases (48\%), pT3 in 6 cases $(29 \%)$ and pT4 in 2 cases $(9 \%)$. The ratio of pT1 and pT2 was higher in cases with extensive intraepithelial spread than without extensive intraepithelial spread $(P=0.040)$. As shown in Table 1, intraepithelial-spreading bile duct carcinoma showed significantly infrequent portal vein or hepatic invasion compared to carcinoma without extensive intraepithelial spread $(P=0.049$ each $)$. There were no differences with respect to frequencies of lymph node metastasis, distant metastasis, invasion to the pancreas, lymphatic vessel invasion, venous invasion, and perineural invasion between carcinoma with and without extensive intraepithelial spread.

\section{Histological Features of Extensive Intraepithelial Spread}

The histological growth type of extensive intraepithelial spread was classifiable into low papillary or flat. The former was characterized by a short fibrovascular stalk that was covered by carcinoma cells (Figure 3a), whereas the latter was characterized by a carcinoma in situ-like spread without folding or fibrovascular stalks (Figure 3b). Seventeen of twenty-one cases of extrahepatic bile duct carcinoma with extensive intraepithelial spread $(81 \%)$ showed low papillary growth in intraepithelial spread. As shown in Table 2, low papillary growth in intraepithelial spread was closely correlated to the gross type of the main tumors. Low papillary growth was commonly observed in cases with main tumors of papillary and nodular types. Notably, all cases having papillary tumors showed low papillary growth in intraepithelial-spreading fields. In contrast, four cases $(19 \%)$ showed flat growth in intraepithelial-spreading areas. Both the cases having the infiltrating type main tumor showed flat growth in intraepithelial spread.

\section{Correlation of Macroscopic and Histological Features of Extensive Intraepithelial Spread}

Macroscopic color pictures of the resected bile duct were available in 12 cases (11 low papillary and 1 flat growths in intraepithelial-spreading fields). We compared histological features of intraepithelial spread with gross appearance. From the gross appearance, the intraepithelial spread could be divided into small granular mucosa and almost normal mucosa (Figure 4). The former showed 
Table 1 Clinicopathological features of extrahepatic bile duct carcinoma with and without extensive intraepithelial spread

\begin{tabular}{|c|c|c|c|}
\hline & $\begin{array}{c}\text { With } \\
\text { intraepithelial } \\
\text { spread }(\mathrm{n}=21)\end{array}$ & $\begin{array}{c}\text { Without } \\
\text { intraepithelial } \\
\text { spread }(\mathrm{n}=96)\end{array}$ & P-value \\
\hline Gender & & & 0.162 \\
\hline Male & $19(90 \%)$ & $75(78 \%)$ & \\
\hline Female & $2(10 \%)$ & $19(22 \%)$ & \\
\hline Age (years) & & & 0.196 \\
\hline$\geq 67$ & $9(43 \%)$ & $56(58 \%)$ & \\
\hline$<67$ & $12(57 \%)$ & $40(42 \%)$ & \\
\hline Location & & & 0.008 \\
\hline Proximal & $13(62 \%)$ & $83(86 \%)$ & \\
\hline Distal & $8(38 \%)$ & $13(14 \%)$ & \\
\hline Gross type & & & $<0.001$ \\
\hline $\begin{array}{l}\text { Papillary or } \\
\text { nodular }\end{array}$ & $19(90 \%)$ & $37(38 \%)$ & \\
\hline Infiltrating & $2(10 \%)$ & $59(61 \%)$ & \\
\hline Histological grade & & & 0.005 \\
\hline $\begin{array}{l}\text { Well- } \\
\text { differentiated }\end{array}$ & $12(57 \%)$ & $25(26 \%)$ & \\
\hline Others & $9(43 \%)$ & $71(74 \%)$ & \\
\hline$P T$ & & & 0.040 \\
\hline $\mathrm{pT} 1$ or pT2 & $13(62 \%)$ & $36(38 \%)$ & \\
\hline pT3 or pT4 & $8(38 \%)$ & $60(62 \%)$ & \\
\hline$P N$ & & & 0.336 \\
\hline pNo & $14(67 \%)$ & $53(55 \%)$ & \\
\hline pN1 & $7(33 \%)$ & $43(45 \%)$ & \\
\hline$P M$ & & & 0.635 \\
\hline pMo & $20(95 \%)$ & $92(96 \%)$ & \\
\hline pM1 & $1(5 \%)$ & $4(4 \%)$ & \\
\hline $\begin{array}{l}\text { Portal vein } \\
\text { invasion }\end{array}$ & & & 0.049 \\
\hline Present & $2(10 \%)$ & $28(29 \%)$ & \\
\hline Absent & $19(90 \%)$ & $68(71 \%)$ & \\
\hline Hepatic invasion & & & 0.049 \\
\hline Present & $2(10 \%)$ & $28(29 \%)$ & \\
\hline Absent & $19(90 \%)$ & $68(71 \%)$ & \\
\hline Pancreatic invasion & & & 0.509 \\
\hline Present & $6(29 \%)$ & $21(22 \%)$ & \\
\hline Absent & $15(71 \%)$ & $75(78 \%)$ & \\
\hline $\begin{array}{l}\text { Lymphatic vessel } \\
\text { invasion }\end{array}$ & & & 0.720 \\
\hline Present & $14(67 \%)$ & $60(63 \%)$ & \\
\hline Absent & $7(33 \%)$ & $36(37 \%)$ & \\
\hline Venous invasion & & & 0.940 \\
\hline Present & $12(57 \%)$ & $54(56 \%)$ & \\
\hline Absent & $9(43 \%)$ & $42(44 \%)$ & \\
\hline Perineural invasion & & & 0.064 \\
\hline Present & $15(71 \%)$ & $84(88 \%)$ & \\
\hline Absent & $6(29 \%)$ & $12(12 \%)$ & \\
\hline
\end{tabular}

multiple small granular lesions covered by a reddish and edematous mucosa, whereas the latter was not apparently different from the surrounding non-
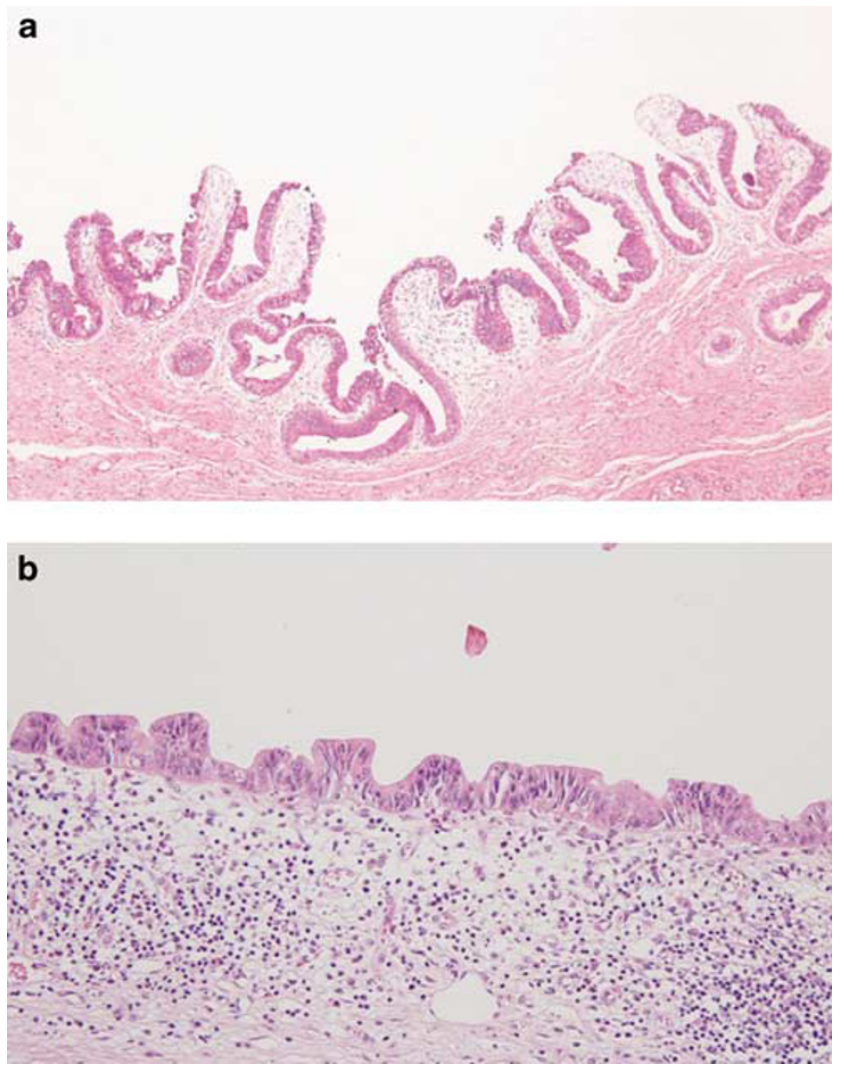

Figure 3 Two histological patterns of extensive intraepithelial spread of carcinoma. (a) Low-papillary pattern. (b) Flat pattern. $(\mathrm{H} \& \mathrm{E}$, original magnification $\times 40)$.

Table 2 Correlation of types of main tumors and extensive intraepithelial spread

\begin{tabular}{lrrr}
\hline & $\mathrm{n}$ & \multicolumn{2}{c}{ Intraepithelial spread } \\
\cline { 3 - 4 } & & Low papillary & Flat \\
\hline Main tumor & & & \\
Papillary type & 11 & 11 & 0 \\
Nodular type & 8 & 6 & 2 \\
Infiltrating type & 2 & 0 & 2 \\
\hline
\end{tabular}

neoplastic mucosa. Histological growth types of intraepithelial spread were closely correlated with macroscopic appearance. That is, all of 11 cases with histologically low papillary growth corresponded to macroscopic small granular mucosa, whereas one case of flat growth showed an almost normal biliary mucosa.

\section{Immunostaining of p53, CEA, MUC1, and Mesothelin}

As shown in Table 3, p53 expression in the main tumors was observed in seven cases (33\%). Among them, five cases (24\%) also showed p53 expression in intraepithelial-spreading area (Figure 5). CEA 

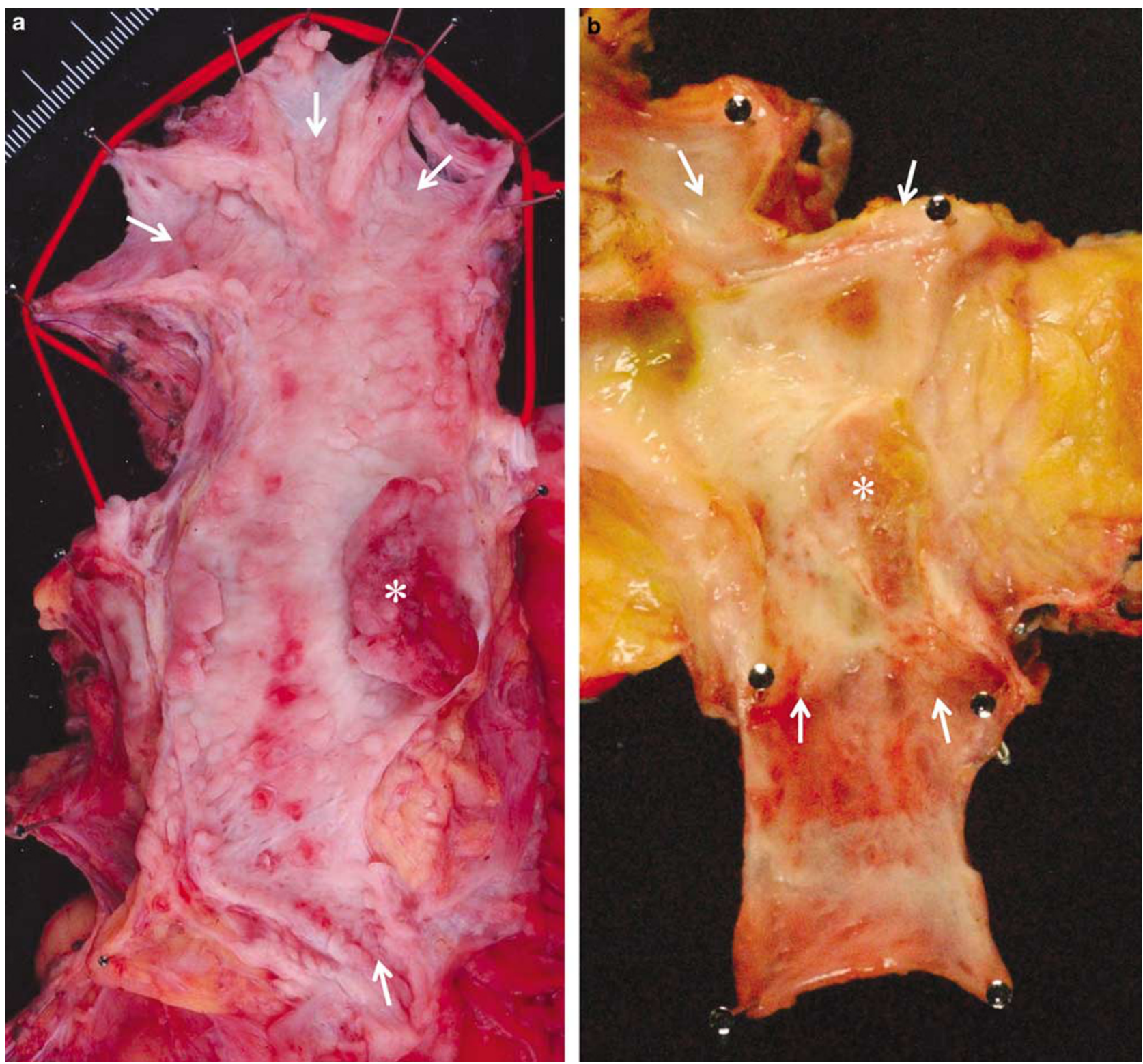

Figure 4 Gross images of extrahepatic bile duct carcinoma with extensive intraepithelial spread. (a) Intraepithelial lesion shows a small granular and reddish mucosa (arrows). (b) Intraepithelial lesion indicated by arrows could not be identified by gross image. ( ${ }^{*}$, main tumors)

expression was observed in the main tumors of 16 cases $(67 \%), 12$ cases of which also expressed CEA in intraepithelial-spreading area (57\%) (Figure 5). MUC1 was expressed in intraepithelial-spreading lesions in 13 cases (62\%), and in main tumors in 17 cases (81\%) (Figure 5). Interestingly, two cases showed MUC1 expression only in intraepithelialspreading areas. Mesothelin was expressed in the main tumor in 6 of 21 cases (29\%). Only one case had mesothelin expression in both the main tumor and intraepithelial-spreading area. Interestingly, the intraepithelial-spreading lesion in this case consisted of a flat proliferation of high-grade carcinoma cells (Figure 6). At least one of p53, CEA, and MUC1 was expressed in the extensive intraepithelial spread in 16 cases $(76 \%)$.

\section{Postoperative Prognosis}

A postoperative follow-up study of 117 patients with extrahepatic bile duct carcinoma disclosed that the presence of extensive intraepithelial spread was associated with a better postoperative prognosis $(P=0.009)$. (Figure 7). Out of 21 patients with extensive intraepithelial spread, 10 showed recurrence, 8 were alive without recurrence, 1 died early postoperatively, and 2 died of other diseases. Among the eight patients alive without recurrence, intraepithelial carcinoma was present at the bile duct stumps on histological examinations in two cases. Those patients were disease-free for 18 and 35 months. Among the 10 cases of recurrence, recurrent tumors were observed in the liver (five cases) and 
the remnant bile duct (anastomotic site) (three cases). Detailed information on recurrence was not available in two cases. Three cases with anastomotic recurrence had carcinoma in situ at the bile duct stumps ( $\leq 3 \mathrm{~mm}$ from the surgical margin). Diseasefree periods in those cases were 54, 66, and 130 months, and survival periods were 75, 70, and 143 months, respectively.

Table 3 Expression of p53, CEA, MUC1, and mesothelin in main tumors and extensive intraepithelial spread

\begin{tabular}{|c|c|c|c|c|c|c|c|c|}
\hline & \multicolumn{8}{|c|}{ Extensive intraepithelial spread } \\
\hline & \multicolumn{2}{|c|}{ p53 } & \multicolumn{2}{|c|}{$C E A$} & \multicolumn{2}{|c|}{ MUC1 } & \multicolumn{2}{|c|}{ Mesothelin } \\
\hline & - & + & - & + & - & + & - & + \\
\hline \multicolumn{9}{|c|}{ Main tumor } \\
\hline- & 14 & 0 & 5 & 0 & 2 & 2 & 15 & 0 \\
\hline+ & 2 & 5 & 4 & 12 & 6 & 11 & 5 & 1 \\
\hline
\end{tabular}
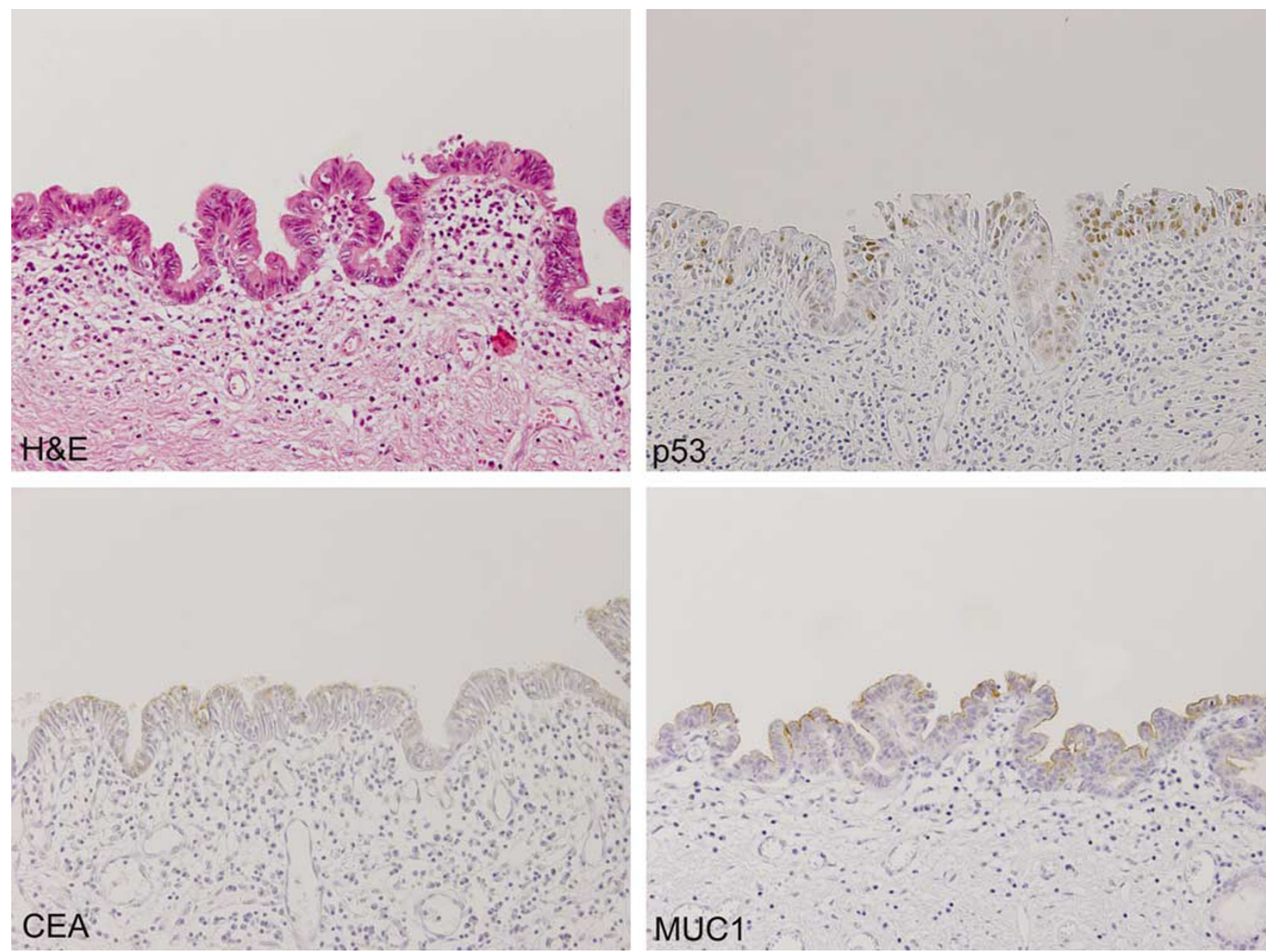

\section{Discussion}

Intraepithelial carcinoma adjacent to the main tumor is reportedly found in about $10-75 \%$ of cases of extrahepatic bile duct carcinoma. ${ }^{8-11}$ Some such cases show an extensive intraepithelial spread of carcinoma cells around the main tumor, and this type of growth has also been called superficial spread.,12-14 In this study, intraepithelial spread adjacent to the main tumors was found in 46 of 117 cases $(39 \%)$. About half of those cases (18\%) showed a spread $\geq 20 \mathrm{~mm}$ around the main tumor. Extensive intraepithelial spread $(\geq 20 \mathrm{~mm}$ intraepithelial spread) seems less common in extrahepatic bile duct carcinoma, but may cause the surgical margin to test positive for carcinoma in situ. One has to carefully examine the extent of superficial spread in cases with papillary or nodular main tumor, because $90 \%$ of cases with extensive intraepithelial spread are papillary or nodular tumors.

Most of the extensive intraepithelial spread associated with papillary or nodular tumors showed 

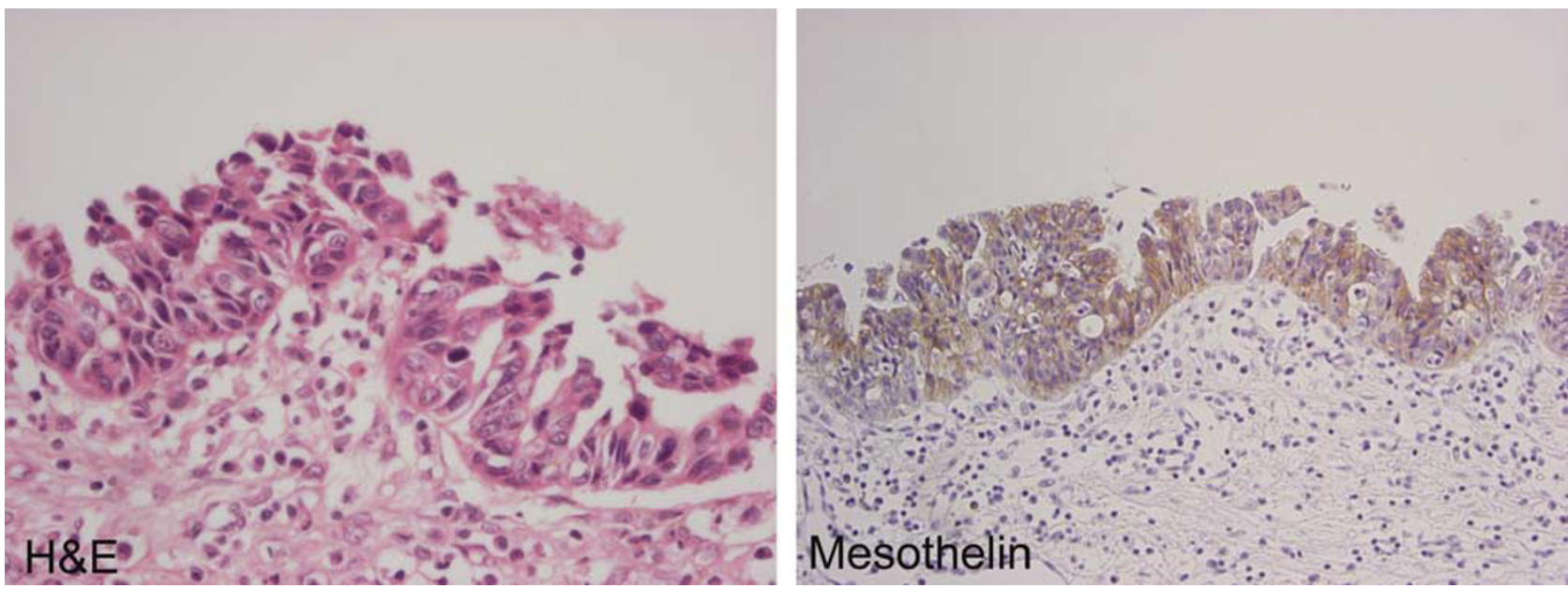

Figure 6 Immunostaining of mesothelin in a case of extrahepatic bile duct carcinoma with extensive intraepithelial spread. Intraepithelial carcinoma consists of high-grade carcinoma cells. (H\&E, original magnification $\times 400$ ). Intraepithelial carcinoma shows cytoplasmic expression of mesothelin. (original magnification $\times 200$ ).

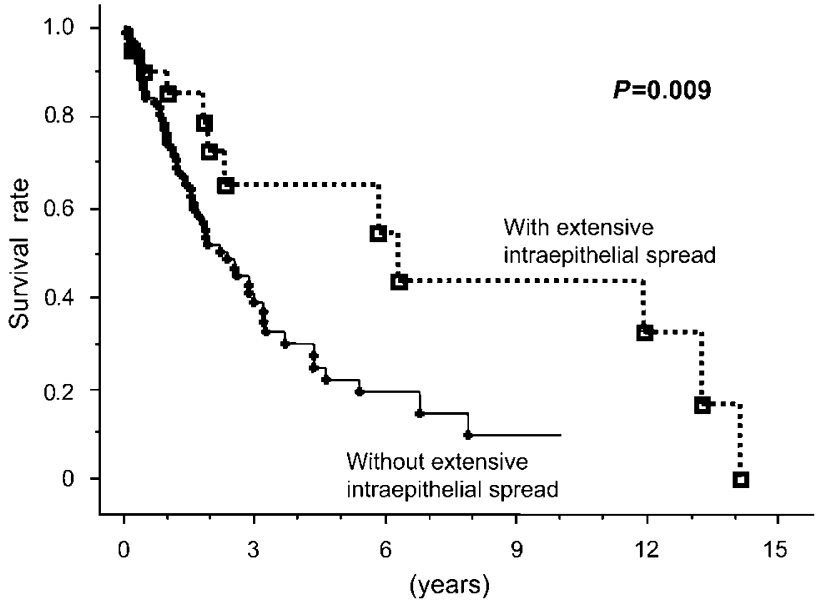

Figure 7 Postoperative survival curves of patients with extrahepatic bile duct carcinoma with and without extensive intraepithelial spread evaluated by the Kaplan-Meier method. Patients with extensive intraepithelial spread have a more favorable prognosis than those without extensive intraepithelial spread $(P=0.009)$.

a histologically low papillary pattern and grossly small granular mucosa. One may detect the intraepithelial spread with a cholangioscope, because mucosal findings have been examined using percutaneous transhepatic cholangioscopy or percutaneous transoral cholangioscopy. ${ }^{15-17}$ In addition, such mucosae are sometimes recognized as 'irregularity of the wall of bile ducts' on cholangiograms. ${ }^{15}$ On the other hand, extrahepatic bile duct carcinoma of infiltrating type and a subset of nodular type of main tumors showed flat-type intraepithelial spread. It seems difficult to detect the flat type of extensive intraepithelial spread with a cholangioscope or cholangiography. Biopsies from the biliary mucosa seem mandatory for preoperative detection of flat-type intraepithelial spread.
The pathogenesis of extensive intraepithelial spread is an interesting issue. Three possibilities have been raised. The first is that the carcinoma in situ spreads extensively before starting invasive growth. Second, carcinoma cells spread from the surface of the invasive tumor. The last possibility is epithelial cancerization, which means that invasive cancer involves the epithelium again and spreads laterally. We do not have sufficient data to make a conclusion, but we speculated that extensive intraepithelial spread can not be explained by a single phenomenon. In our previous study, we examined p53 expression in BilIN not associated with invasive cancer. ${ }^{18}$ Only $11 \%$ of BilIN-3 corresponding to carcinoma in situ were positive for p53, whereas $82 \%$ of invasive cholangiocarcinomas expressed p53. ${ }^{18}$ That is, p53 is usually expressed at the late phase of carcinogenesis in cases of bile duct cancer. In this study, two patients had p53 expression only in the main tumor and not in the intraepithelialspreading area. In those cases, the carcinoma cells might have spread laterally before any invasive growth. Hassan et $a l^{19}$ reported that almost all cases of extrahepatic bile duct carcinoma, pancreatic adenocarcinoma, and ampullar adenocarcinoma were immunohistochemically positive for mesothelin; however, most pancreatic intraepithelial neoplasias, the non-neoplastic pancreatic tissue adjacent to adenocarcinomas, and the ducts of chronic pancreatitis were negative. It was suggested that mesothelin could become a marker for invasive cancer in the pancreatobiliary system. In the present study, only one case was immunohistochemically positive for mesothelin in both the main tumor and intraepithelial-spreading lesion. Interestingly, carcinoma cells in the intraepithelial-spreading area, in this case, were histologically high grade. The extensive intraepithelial spread in this case might have involved epithelial cancerization by invasive cancer. 
The present study showed that the main tumors in cases of extrahepatic bile duct carcinoma with extensive intraepithelial spread were histologically characterized by greater differentiation and less invasive growth (pT1 or pT2) in comparison to those without extensive intraepithelial spread. Extrahepatic bile duct carcinomas with extensive intraepithelial spread are less invasive, and rather proliferate locally, showing papillary or nodular lesions. Interestingly, two cases with the infiltrating main tumor showed flat-type intraepithelial spread. Pathological processes of extensive intraepithelial spread of low papillary and flat types might be different, and the two types of spreading might reflect different pathologic significances. Further pathological and genetic analyses seem mandatory to elucidate this issue.

It is sometimes difficult to evaluate intraepithelial dysplastic epithelium at the surgical margin, because secondary histological changes due to cholangitis or bile duct stents might be superimposed. ${ }^{10}$ However, it is important to decide whether or not intraepithelial carcinoma is present at the surgical margin, because this lesion might result in a late anastomotic recurrence. Diagnosis of intraepithelial dysplastic lesion is mostly based on histological findings themselves. But, immunostaining might support the pathological diagnosis. Murata et $a l^{20}$ reported that immunostaining of p53 was useful to diagnose cancerous lesions by cholangioscopic biopsy. Terada et $a l^{21}$ reported that CEA was not expressed in hyperplastic biliary epithelium, but expressed in most cases of invasive adenocarcinoma and carcinoma in situ. We previously reported that MUC1 was not expressed in non-neoplastic biliary epithelium in hepatolithiasis. ${ }^{22}$ In this study, 16 of 21 cases $(76 \%)$ of extrahepatic bile duct carcinoma with extensive intraepithelial spread were positive for at least one of p53, CEA, and MUC1 in the intraepithelial-spreading area. Immunostaining of p53, CEA, and MUC1 might be useful for evaluation of the extent of intraepithelial spread.

Previously, we examined pathological features of papillary and non-papillary bile duct carcinomas, and pancreatic intraductal papillary mucinous neoplasm. ${ }^{23}$ Papillary bile duct carcinoma had pathological characteristics, such as prominent intraductal growth, the existence of three types of tumor cells (pancreatobiliary, intestinal, and gastric types), possible progression to two types of invasive lesions (tubular adenocarcinoma and mucinous carcinoma), and the common expression of MUC2, CDX2 and cytokeratin $20 .^{23}$ Those characteristics of papillary bile duct carcinoma were different from those of conventional non-papillary carcinoma, rather closely resembled those of pancreatic intraductal papillary mucinous neoplasm. We concluded that papillary bile duct carcinoma could be included in a tumor entity named 'intraductal papillary neoplasm of the bile duct', as the biliary counterpart of pancreatic intraductal papillary mucinous neo- plasm. From this viewpoint, 11 cases having papillary main tumor examined in this study can be estimated as intraductal papillary neoplasm of the bile duct. All of those cases were found to correspond to the pancreatobiliary type described in the pancreas. ${ }^{24,25}$ Different from biliary papillomatosis (another member of biliary intraductal papillary neoplasm), any cases examined in this study did not have adenoma component or multiple lesions. Extensive intraepithelial spread seems to be a more common phenomenon of intraductal papillary neoplasm of the bile duct compared to conventional non-papillary bile duct carcinoma.

Interestingly, the postoperative follow-up study disclosed that extensive intraepithelial spread itself was a favorable prognostic factor. This result was probably due to more differentiated histological grade, less invasiveness, and localized growth in intraepithelial-spreading bile duct carcinoma. However, 10 of 21 cases with extensive intraepithelial spread showed recurrent carcinoma. Notably, three cases had late anastomotic recurrence, and the mean disease free periods in those cases were about 7 years. This result suggests that intraepithelialspreading lesions have a low malignant nature, and the presence of intraepithelial carcinoma within the remnant bile duct could cause late recurrence. One should make efforts for total resection of the tumor including extensive intraepithelial spread. However, extended resections of the liver or pancreas increase surgical invasiveness in patients, especially the elderly. Careful selection of the surgical procedure taking clinical factors into consideration is necessary, because the remnant intraepithelial carcinoma is unlikely to result in early fatal recurrence.

In conclusion, extensive intraepithelial spread was found in $18 \%$ of surgically resected extrahepatic bile duct carcinoma cases. Intraepithelialspreading bile duct carcinoma is pathologically characterized by a papillary or nodular-type main tumor, more differentiated histological type, and lesser invasiveness. The presence of extensive intraepithelial spread is one of the factors associated with a favorable prognosis in cases of extrahepatic bile duct carcinoma, but might be a cause of late local recurrence when left in the remnant bile duct.

\section{References}

1 Sakamoto E, Nimura Y, Hayakawa N, et al. The pattern of infiltration at the proximal border of hilar bile duct carcinoma: a histologic analysis of 62 resected cases. Ann Surg 1998;227:405-411.

2 Malhi H, Gores GJ. Cholangiocarcinoma: modern advances in understanding a deadly old disease. J Hepatol 2006;45:856-867.

3 Wakai T, Shirai Y, Moroda T, et al. Impact of ductal resection margin status on long-term survival in 
patients undergoing resection for extrahepatic cholangiocarcinoma. Cancer 2005;103:1210-1216.

4 Nakanishi Y, Kondo S, Hirano S, et al. Recurrence of mucosal carcinoma of the bile duct, with superficial flat spread, 12 years after operation. J Hepatobiliary Pancreat Surg 2006;13:355-358.

5 Sasaki T, Kondo S, Ambo Y, et al. Local recurrence at hepaticojejunostomy 9 years after resection of bile duct cancer with superficial flat spread. J Hepatobiliary Pancreat Surg 2006;13:458-462.

6 Zen Y, Adsay NV, Bardadin K, et al. Biliary intraepithelial neoplasia: an international interobserver agreement study and proposal for diagnostic criteria. Mod Pathol 2007;20:701-709.

7 Sobin LH, Wittekind CH. TNM Classification of Malignant Tumors, 6th edn. Wiley-Liss: New York 2000, pp 87-89.

8 Laitio M. Carcinoma of extrahepatic bile ducts. A histopathologic study. Pathol Res Pract 1983;178: 67-72.

9 Suzuki M, Takahashi T, Ouchi K, et al. The development and extension of hepatohilar bile duct carcinoma. A three-dimensional tumor mapping in the intrahepatic biliary tree visualized with the aid of a graphics computer system. Cancer 1989;64: 658-666.

10 Albores-Saavedra J, Henson DE, Klimstra DA. Tumors of the Gallbadder, Etrahepatic Bile Ducts and Ampulla of Vater, Atras of Tumor Pathology, 3rd series, Fascile 27. Armed Forces Institute of Pathology: Washington, DC, 2000, pp 191-215.

11 Davis RI, Sloan JM, Hood JM, et al. Carcinoma of the extrahepatic biliary tract: a clinicopathological and immunohistochemical study. Histopathology 1988;12:623-631.

12 Kato M, Nimura Y, Kamiya J, et al. Carcinoma of the common bile duct with superficial spread to the intrahepatic segmental bile ducts: a case report. Am Surg 1997;63:943-947.

13 Abe M, Kondo S, Hirano S, et al. Superficially spreading cholangiocarcinoma. Int J Gastrointest Cancer 2005;35:89-94.

14 Iwahashi N, Hayakawa N, Yamamoto H, et al. Mucosal bile duct carcinoma with superficial spread. J Hepatobiliary Pancreat Surg 1998;5:221-225.
15 Nimura Y. Staging of biliary carcinoma: cholangiography and cholangioscopy. Endoscopy 1993;25:76-80.

16 Sato $\mathrm{M}$, Inoue $\mathrm{H}$, Ogawa $\mathrm{S}$, et al. Limitations of percutaneous transhepatic cholangioscopy for the diagnosis of the intramural extension of bile duct carcinoma. Endoscopy 1998;30:281-288.

17 Nimura Y, Kamiya J. Cholangioscopy. Endoscopy 1998;30:182-188.

18 Nakanishi Y, Zen Y, Kondo S, et al. Expression of cell cycle-related molecules in biliary premalignant lesions: biliary intraepithelial neoplasia and biliary intraductal papillary neoplasm. Hum Pathol, in press.

19 Hassan R, Laszik ZG, Lerner M, et al. Mesothelin is overexpressed in pancreaticobiliary adenocarcinomas but not in normal pancreas and chronic pancreatitis. Am J Clin Pathol 2005;124:838-845.

20 Murata T, Nagasaka T, Kamiya J, et al. P53 labeling index in cholangioscopic biopsies is useful for determining spread of bile duct carcinomas. Gastrointest Endosc 2002;56:688-695.

21 Terada T, Nakanuma Y. Cell kinetic analyses and expression of carcinoembryonic antigen, carbohydrate antigen 19-9 and DU-PAN-2 in hyperplastic, preneoplastic and neoplastic lesions of intrahepatic bile ducts in livers with hepatoliths. Virchows Arch A Pathol Anat Histopathol 1992;420:327-335.

22 Zen Y, Sasaki M, Fujii T, et al. Different expression patterns of mucin core proteins and cytokeratins during intrahepatic cholangiocarcinogenesis from biliary intraepithelial neoplasia and intraductal papillary neoplasm of the bile duct-an immunohistochemical study of 110 cases of hepatolithiasis. J Hepatol 2006;44:350-358.

23 Zen Y, Fujii T, Itatsu K, et al. Biliary papillary tumors share pathological features with intraductal papillary mucinous neoplasm of the pancreas. Hepatology 2006;44:1333-1343

24 Andrejevic-Blant S, Kosmahl M, Sipos B, et al. Pancreatic intraductal papillary-mucinous neoplasms: a new and evolving entity. Virchows Arch 2007;451:863-869.

25 Furukawa T, Klöppel G, Adsay NV, et al. Classification of types of intraductal papillary-mucinous neoplasm of the pancreas: a consensus study. Virchows Arch 2005;447:794-799. 